\title{
Refined dual stable Grothendieck polynomials and generalized Bender-Knuth involutions
}

\author{
Pavel Galashin, Darij Grinberg, and Gaku Liu
}

Massachusetts Institute of Technology, USA

\begin{abstract}
The dual stable Grothendieck polynomials are a deformation of the Schur functions, originating in the study of the $K$-theory of the Grassmannian. We generalize these polynomials by introducing a countable family of additional parameters such that the generalization still defines symmetric functions. We outline two self-contained proofs of this fact, one of which constructs a family of involutions on the set of reverse plane partitions generalizing the Bender-Knuth involutions on semistandard tableaux, whereas the other classifies the structure of reverse plane partitions with entries 1 and 2 .

Résumé. Les polynômes de Grothendieck stables duaux sont une déformation des fonctions de Schur provenant de l'étude de la K-théorie de la Grassmannienne. Nous généralisons ces polynômes en introduisant une famille dénombrable de paramètres additionnels de sorte que cette généralisation définisse encore des fonctions symétriques. Nous présentons deux preuves auto-suffisantes de ce fait, dont l'une construit une famille d'involutions de l'ensemble des partitions planes inversées généralisant les involutions de Bender-Knuth sur les tableaux semi-standards, tandis que l'autre classifie la structure des partitions planes avec entrées 1 et 2.
\end{abstract}

Keywords. symmetric functions, reverse plane partitions, Bender-Knuth involutions

\section{Introduction}

Thomas Lam and Pavlo Pylyavskyy, in [LamPy107, §9.1], (and earlier Mark Shimozono and Mike Zabrocki in unpublished work of 2003) studied dual stable Grothendieck polynomials, a deformation (in a sense) of the Schur functions. Let us briefly recount their definition.

Let $\lambda / \mu$ be a skew partition. The Schur function $s_{\lambda / \mu}$ is a multivariate generating function for the semistandard tableaux of shape $\lambda / \mu$. In the same vein, the dual stable Grothendieck polynomial $g_{\lambda / \mu}$ is a generating function for the reverse plane partitions of shape $\lambda / \mu$; these, unlike semistandard tableaux, are only required to have their entries increase weakly down columns (and along rows). More precisely, $g_{\lambda / \mu}$ is a formal power series in countably many commuting indeterminates $x_{1}, x_{2}, x_{3}, \ldots$ defined by

$$
g_{\lambda / \mu}=\sum_{\substack{T \text { is a reverse plane } \\ \text { partition of shape } \lambda / \mu}} \mathbf{x}^{\operatorname{ircont}(T)},
$$

where $\mathrm{x}^{\mathrm{ircont}(T)}$ is the monomial $x_{1}^{a_{1}} x_{2}^{a_{2}} x_{3}^{a_{3}} \cdots$ whose $i$-th exponent $a_{i}$ is the number of columns (rather than cells) of $T$ containing the entry $i$. As proven in [LamPyl07, §9.1], this power series $g_{\lambda / \mu}$ is a symmetric function (albeit, unlike $s_{\lambda / \mu}$, an inhomogeneous one in general). Lam and Pylyavskyy connect the 
$g_{\lambda / \mu}$ to the (more familiar) stable Grothendieck polynomials $G_{\lambda / \mu}$ (via a duality between the symmetric functions and their completion, which explains the name of the $g_{\lambda / \mu}$; see [LamPyl07, §9.4]) and to the $K$-theory of Grassmannians ([LamPyl07, §9.5]).

We devise a common generalization of the dual stable Grothendieck polynomial $g_{\lambda / \mu}$ and the classical skew Schur function $s_{\lambda / \mu}$. Namely, if $t_{1}, t_{2}, t_{3}, \ldots$ are countably many indeterminates, then we set

$$
\widetilde{g}_{\lambda / \mu}=\sum_{\substack{T \text { is a reverse plane } \\ \text { partition of shape } \lambda / \mu}} \mathbf{t}^{\operatorname{ceq}(T)} \mathbf{x}^{\operatorname{ircont}(T)},
$$

where $\mathbf{t}^{\mathrm{ceq}(T)}$ is the product $t_{1}^{b_{1}} t_{2}^{b_{2}} t_{3}^{b_{3}} \ldots$ whose $i$-th exponent $b_{i}$ is the number of cells in the $i$-th row of $T$ whose entry equals the entry of their neighbor cell directly below them. This $\widetilde{g}_{\lambda / \mu}$ becomes $g_{\lambda / \mu}$ when all the $t_{i}$ are set to 1 , and becomes $s_{\lambda / \mu}$ when all the $t_{i}$ are set to 0 .

Our main result, Theorem 3.3 states that $\widetilde{g}_{\lambda / \mu}$ is a symmetric function (in the $x_{1}, x_{2}, x_{3}, \ldots$ ).

We outline two proofs this result (thus obtaining a new proof of [LamPyl07, Theorem 9.1]), first using an elaborate generalization of the classical Bender-Knuth involutions to reverse plane partitions, and then for a second time by analyzing the structure of reverse plane partitions whose entries lie in $\{1,2\}$. The second proof reflects back on the first, in particular providing an alternative definition of the generalized Bender-Knuth involutions constructed in the first proof, and showing that these involutions are (in a sense) "the only reasonable choice". The full proofs can be found in [GGL15].

\subsection{Acknowledgments}

We owe our familiarity with dual stable Grothendieck polynomials to Richard Stanley. We thank Alexander Postnikov for providing context and motivation, and Thomas Lam and Pavlo Pylyavskyy for interesting conversations.

\section{Notations and definitions}

Let us begin by defining our notations (including some standard conventions from algebraic combinatorics).

\subsection{Partitions and tableaux}

We set $\mathbb{N}=\{0,1,2, \ldots\}$ and $\mathbb{N}_{+}=\{1,2,3, \ldots\}$.

A sequence $\alpha=\left(\alpha_{1}, \alpha_{2}, \alpha_{3}, \ldots\right)$ of nonnegative integers is called a weak composition if the sum of its entries (denoted $|\alpha|$ ) is finite. We shall always write $\alpha_{i}$ for the $i$-th entry of a weak composition $\alpha$.

A partition is a weak composition $\left(\alpha_{1}, \alpha_{2}, \alpha_{3}, \ldots\right)$ satisfying $\alpha_{1} \geq \alpha_{2} \geq \alpha_{3} \geq \cdots$. As usual, we often omit trailing zeroes when writing a partition (e.g., the partition $(5,2,1,0,0,0, \ldots)$ can thus be written as $(5,2,1))$.

We identify each partition $\lambda$ with the subset $\left\{(i, j) \in \mathbb{N}_{+}^{2} \mid j \leq \lambda_{i}\right\}$ of $\mathbb{N}_{+}^{2}$ (called the Young diagram of $\lambda$ ). We draw this subset as a Young diagram (which is a left-aligned table of empty boxes, where the box $(1,1)$ is in the top-left corner while the box $(2,1)$ is directly below it; this is the English notation, also known as the matrix notation); see [Fulton97] for the detailed definition.

A skew partition $\lambda / \mu$ is a pair $(\lambda, \mu)$ of partitions satisfying $\mu \subseteq \lambda$ (as subsets of $\mathbb{N}_{+}^{2}$ ). In this case, we shall also often use the notation $\lambda / \mu$ for the set-theoretic difference of $\lambda$ and $\mu$. 


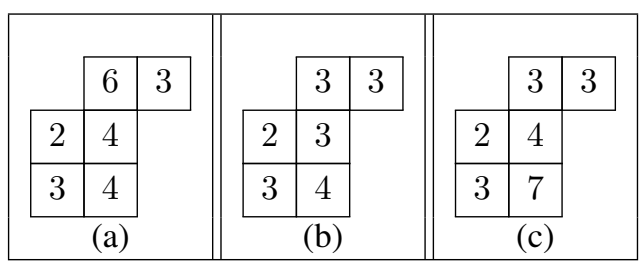

Fig. 1: Fillings of $(3,2,2) /(1)$ : (a) is not an rpp as it has a 4 below a 6 , (b) is an rpp but not a semistandard tableau as it has a 3 below a $3,(\mathrm{c})$ is a semistandard tableau (and hence also an rpp).

If $\lambda / \mu$ is a skew partition, then a filling of $\lambda / \mu$ means a map $T: \lambda / \mu \rightarrow \mathbb{N}_{+}$. It is visually represented by drawing $\lambda / \mu$ and filling each box $c$ with the entry $T(c)$. Three examples of a filling can be found on Figure 1.

A filling $T: \lambda / \mu \rightarrow \mathbb{N}_{+}$of $\lambda / \mu$ is called a reverse plane partition of shape $\lambda / \mu$ if its values increase weakly in each row of $\lambda / \mu$ from left to right and in each column of $\lambda / \mu$ from top to bottom. If, in addition, the values of $T$ increase strictly down each column, then $T$ is called a semistandard tableau of shape $\lambda / \mu$. (See Fulton's [Fulton97] for an exposition of properties and applications of semistandard tableaux.) We denote the set of all reverse plane partitions of shape $\lambda / \mu$ by $\operatorname{RPP}(\lambda / \mu)$. We abbreviate reverse plane partitions as rpps.

Examples of an rpp, of a non-rpp and of a semistandard tableau can be found on Figure 1

\subsection{Symmetric functions}

A symmetric function is defined to be a bounded-degree power series in countably many indeterminates $x_{1}, x_{2}, x_{3}, \ldots$ over $\mathbb{Z}$ that is invariant under (finite) permutations of $x_{1}, x_{2}, x_{3}, \ldots$

The symmetric functions form a ring, which is called the ring of symmetric functions and denoted by $\Lambda$. (In [LamPy107] this ring is denoted by Sym, while the notation $\Lambda$ is reserved for the set of all partitions.) Much research has been done on symmetric functions and their relations to Young diagrams and tableaux; see [Stan99, Chapter 7], [Macdon95] and [GriRei15, Chapter 2] for expositions.

Given a filling $T$ of a skew partition $\lambda / \mu$, its content is a weak composition cont $(T)=\left(r_{1}, r_{2}, r_{3}, \ldots\right)$, where $r_{i}=\left|T^{-1}(i)\right|$ is the number of entries of $T$ equal to $i$. For a skew partition $\lambda / \mu$, we define the Schur function $s_{\lambda / \mu}$ to be the formal power series

$$
s_{\lambda / \mu}\left(x_{1}, x_{2}, \ldots\right)=\sum_{\substack{T \text { is a semistandard } \\ \text { tableau of shape } \lambda / \mu}} \mathbf{x}^{\operatorname{cont}(T)} .
$$

Here, for every weak composition $\alpha=\left(\alpha_{1}, \alpha_{2}, \alpha_{3}, \ldots\right)$, we define a monomial $\mathbf{x}^{\alpha}$ to be $x_{1}^{\alpha_{1}} x_{2}^{\alpha_{2}} x_{3}^{\alpha_{3}} \cdots$. These Schur functions are symmetric:

Proposition 2.1. We have $s_{\lambda / \mu} \in \Lambda$ for every skew partition $\lambda / \mu$.

This result appears, e.g., in [Stan99, Theorem 7.10.2] and [GriRei15, Proposition 2.11]; it is commonly proven bijectively using the so-called Bender-Knuth involutions. We shall recall the definitions of these involutions in Section 5 .

Replacing "semistandard tableau" by "rpp" in the definition of a Schur function in general gives a non-symmetric function. Nevertheless, Lam and Pylyavskyy [LamPyl07, §9] have been able to define symmetric functions from rpps, albeit using a subtler construction instead of the content cont $(T)$. 
Namely, for a filling $T$ of a skew partition $\lambda / \mu$, we define its irredundant content (or, by way of abbreviation, its ircont statistic) as the weak composition $\operatorname{ircont}(T)=\left(r_{1}, r_{2}, r_{3}, \ldots\right)$ where $r_{i}$ is the number of columns (rather than cells) of $T$ that contain an entry equal to $i$. For instance, if $T_{a}, T_{b}$, and $T_{c}$ are the fillings from Figure 1 , then their irredundant contents are

$$
\operatorname{ircont}\left(T_{a}\right)=(0,1,2,1,0,1), \operatorname{ircont}\left(T_{b}\right)=(0,1,3,1), \operatorname{ircont}\left(T_{c}\right)=(0,1,3,1,0,0,1)
$$

(where we omit trailing zeroes), because, for example, $T_{a}$ has one column with a 4 in it (so $\left(\operatorname{ircont}\left(T_{a}\right)\right)_{4}=$ $1)$ and $T_{b}$ contains three columns with a $3\left(\operatorname{so}\left(\operatorname{ircont}\left(T_{b}\right)\right)_{3}=3\right)$.

Notice that if $T$ is a semistandard tableau, then $\operatorname{cont}(T)$ and $\operatorname{ircont}(T)$ coincide.

For the rest of this section, we fix a skew partition $\lambda / \mu$. Now, the dual stable Grothendieck polynomial $g_{\lambda / \mu}$ is defined to be the formal power series

$$
\sum_{\substack{T \text { is an rpp } \\ \text { of shape } \lambda / \mu}} \mathbf{x}^{\operatorname{ircont}(T)} .
$$

Unlike the Schur function $s_{\lambda / \mu}$, it is (in general) not homogeneous, because whenever a column of an rpp $T$ contains an entry several times, the corresponding monomial $x^{\operatorname{ircont}(T)}$ "counts" this entry only once. It is fairly clear that the highest-degree homogeneous component of $g_{\lambda / \mu}$ is $s_{\lambda / \mu}$ (the component of degree $|\lambda|-|\mu|)$. Therefore, $g_{\lambda / \mu}$ can be regarded as an inhomogeneous deformation of the Schur function $s_{\lambda / \mu}$. Lam and Pylyavskyy, in [LamPyl07, §9.1], have shown the following fact:

Proposition 2.2. We have $g_{\lambda / \mu} \in \Lambda$ for every skew partition $\lambda / \mu$.

They prove this proposition using generalized plactic algebras [FomGre06, Lemma 3.1] (and also give a second, combinatorial proof for the case $\mu=\varnothing$ by explicitly expanding $g_{\lambda / \varnothing}$ as a sum of Schur functions).

In the next section, we shall introduce a refinement of these $g_{\lambda / \mu}$, and later we will reprove Proposition 2.2 in a bijective and elementary way.

\section{Refined dual stable Grothendieck polynomials}

\subsection{Definition}

Let $\mathbf{t}=\left(t_{1}, t_{2}, t_{3}, \ldots\right)$ be a sequence of further indeterminates. For any weak composition $\alpha$, we define $\mathbf{t}^{\alpha}$ to be the monomial $t_{1}^{\alpha_{1}} t_{2}^{\alpha_{2}} t_{3}^{\alpha_{3}} \cdots$. By convention, we set $\mathbf{t}^{()}=1$.

If $T$ is a filling of a skew partition $\lambda / \mu$, then a redundant cell of $T$ is a cell of $\lambda / \mu$ whose entry is equal to the entry directly below it. That is, a cell $(i, j)$ of $\lambda / \mu$ is redundant if $(i+1, j)$ is also a cell of $\lambda / \mu$ and $T(i, j)=T(i+1, j)$. Notice that a semistandard tableau is the same thing as an rpp which has no redundant cells.

If $T$ is a filling of $\lambda / \mu$, then we define the column equalities vector (or, by way of abbreviation, the ceq statistic) of $T$ to be the weak composition ceq $(T)=\left(c_{1}, c_{2}, c_{3}, \ldots\right)$ where $c_{i}$ is the number of $j \in \mathbb{N}_{+}$ such that $(i, j)$ is a redundant cell of $T$. Visually speaking, $(\operatorname{ceq}(T))_{i}$ is the number of columns of $T$ whose entry in the $i$-th row equals their entry in the $(i+1)$-th row. For instance, for fillings $T_{a}, T_{b}, T_{c}$ from Figure 1 we have ceq $\left(T_{a}\right)=(0,1), \operatorname{ceq}\left(T_{b}\right)=(1)$, and $\operatorname{ceq}\left(T_{c}\right)=()$, where we again drop trailing zeroes. 
Notice that $|\operatorname{ceq}(T)|$ is the number of redundant cells in $T$, so we have

$$
|\operatorname{ceq}(T)|+|\operatorname{ircont}(T)|=|\lambda / \mu|
$$

for all rpps $T$ of shape $\lambda / \mu$.

Let now $\lambda / \mu$ be a skew partition. We set

$$
\widetilde{g}_{\lambda / \mu}(\mathbf{x} ; \mathbf{t})=\sum_{\substack{T \text { is an rpp } \\ \text { of shape } \lambda / \mu}} \mathbf{t}^{\mathrm{ceq}(T)} \mathbf{x}^{\mathrm{ircont}(T)} .
$$

which is a formal power series in $\mathbf{x}$ with coefficients in $\mathbb{Z}\left[t_{1}, t_{2}, t_{3}, \ldots\right]$.

Let us give some examples of $\widetilde{g}_{\lambda / \mu}$.

Example 3.1. (a) If $\lambda / \mu$ is a single row with $n$ cells, then for each rpp $T$ of shape $\lambda / \mu$ we have $\operatorname{ceq}(T)=(0,0, \ldots)$ and $\operatorname{ircont}(T)=\operatorname{cont}(T)$ (in fact, any rpp of shape $\lambda / \mu$ is a semistandard tableau in this case). Therefore we get

$$
\widetilde{g}_{\lambda / \mu}(\mathbf{x} ; \mathbf{t})=h_{n}(\mathbf{x})=\sum_{a_{1} \leq a_{2} \leq \cdots \leq a_{n}} x_{a_{1}} x_{a_{2}} \cdots x_{a_{n}} .
$$

Here $h_{n}(\mathbf{x})$ is the $n$-th complete homogeneous symmetric function.

(b) If $\lambda / \mu$ is a single column with $n$ cells, then, by 11 , for all $\operatorname{rpps} T$ of shape $\lambda / \mu$ we have $|\operatorname{ceq}(T)|+$ $|\operatorname{ircont}(T)|=n$, so in this case

$$
\widetilde{g}_{\lambda / \mu}(\mathbf{x} ; \mathbf{t})=\sum_{k=0}^{n-1} e_{k}\left(t_{1}, t_{2}, \ldots, t_{n-1}\right) e_{n-k}\left(x_{1}, x_{2}, \ldots\right)=e_{n}\left(t_{1}, t_{2}, \ldots, t_{n-1}, x_{1}, x_{2}, \ldots\right),
$$

where $e_{i}\left(\xi_{1}, \xi_{2}, \xi_{3}, \ldots\right)$ denotes the $i$-th elementary symmetric function in the indeterminates $\xi_{1}, \xi_{2}, \xi_{3}, \ldots$

The power series $\widetilde{g}_{\lambda / \mu}$ generalize the power series $g_{\lambda / \mu}$ and $s_{\lambda / \mu}$ studied before. The following proposition is clear:

Proposition 3.2. Let $\lambda / \mu$ be a skew partition.

(a) Specifying $\mathbf{t}=(1,1,1, \ldots)$ yields $\widetilde{g}_{\lambda / \mu}(\mathbf{x} ; \mathbf{t})=g_{\lambda / \mu}(\mathbf{x})$.

(b) Specifying $\mathbf{t}=(0,0,0, \ldots)$ yields $\widetilde{g}_{\lambda / \mu}(\mathbf{x} ; \mathbf{t})=s_{\lambda / \mu}(\mathbf{x})$.

\subsection{The symmetry statement}

Our main result is now the following:

Theorem 3.3. Let $\lambda / \mu$ be a skew partition. Then $\widetilde{g}_{\lambda / \mu}(\mathbf{x} ; \mathbf{t})$ is symmetric in $\mathbf{x}$.

Here, "symmetric in $\mathbf{x}$ " means "invariant under all finite permutations of the indeterminates $x_{1}, x_{2}, x_{3}, \ldots$ " (while $t_{1}, t_{2}, t_{3}, \ldots$ remain unchanged).

Clearly, Theorem 3.3 implies the symmetry of $g_{\lambda / \mu}$ and $s_{\lambda / \mu}$ due to Proposition 3.2 .

We shall prove Theorem 3.3 bijectively. The core of our proof will be the following restatement of Theorem 3.3. 
Theorem 3.4. Let $\lambda / \mu$ be a skew partition and let $i \in \mathbb{N}_{+}$. Then, there exists an involution $\mathbf{B}_{i}$ : $\operatorname{RPP}(\lambda / \mu) \rightarrow \operatorname{RPP}(\lambda / \mu)$ which preserves the ceq statistics and acts on the ircont statistic by the transposition of its $i$-th and $(i+1)$-th entries.

This involution $\mathbf{B}_{i}$ is a generalization of the $i$-th Bender-Knuth involution defined for semistandard tableaux (see, e.g., [GriRei15, proof of Proposition 2.11]), but its definition is more complicated than that of the latter. Defining it and proving its properties takes a significant part of the full paper.

\subsection{Reduction to 12-rpps}

Fix a skew partition $\lambda / \mu$. We shall make one further simplification before we step to the actual proof of Theorem 3.4 We define a 12-rpp to be an rpp whose entries all belong to the set $\{1,2\}$. We let $\operatorname{RPP}^{12}(\lambda / \mu)$ be the set of all 12-rpps of shape $\lambda / \mu$.

Lemma 3.5. There exists an involution $\mathbf{B}: \operatorname{RPP}^{12}(\lambda / \mu) \rightarrow \operatorname{RPP}^{12}(\lambda / \mu)$ which preserves the ceq statistic and switches the number of columns containing a 1 with the number of columns containing a 2 (that is, switches the first two entries of the ircont statistic).

It is straightforward to see that this Lemma implies Theorem 3.4

\section{Construction of $\mathbf{B}$}

In this section we are going to sketch the definition of $\mathbf{B}$ and state some of its properties. For the whole Section 4 we shall be working in the situation of Lemma 3.5. In particular, we fix a skew partition $\lambda / \mu$.

A 12-table means a filling $T: \lambda / \mu \rightarrow\{1,2\}$ of $\lambda / \mu$ such that the entries of $T$ are weakly increasing down columns. (We do not require them to be weakly increasing along rows.) Every column of a 12-table is a sequence of the form $(1,1, \ldots, 1,2,2, \ldots, 2)$. We say that such a sequence is

- 1-pure if it is nonempty and consists purely of 1 's,

- 2-pure if it is nonempty and consists purely of 2's,

- mixed if it contains both 1's and 2's.

Definition 4.1. For a 12-table $T$, we define flip $(T)$ to be the 12-table obtained from $T$ by changing each column of $T$ as follows:

- If this column is 1-pure, we replace all its entries by 2's (so that it becomes 2-pure).

Otherwise, if this column is 2-pure, we replace all its entries by 1's (so that it becomes 1-pure).

Otherwise (i.e., if this column is mixed or empty), we do not change it.

If $T$ is a 12-rpp then flip $(T)$ need not be a 12-rpp, because it can contain a 2 to the left of a 1 in some row. We say that a positive integer $k$ is a descent of a 12-table $P$ if there is a 2 in the column $k$ and there is a 1 to the right of it in the column $k+1$. We will encounter three possible kinds of descents depending on the types of columns $k$ and $k+1$ :

(M1) The $k$-th column of $P$ is mixed and the $(k+1)$-th column of $P$ is 1-pure.

(2M) The $k$-th column of $P$ is 2-pure and the $(k+1)$-th column of $P$ is mixed. 


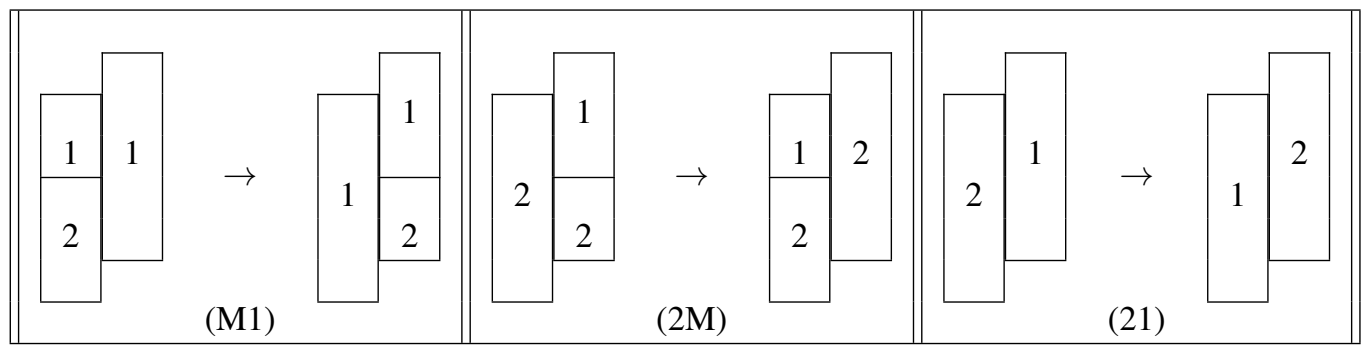

Fig. 2: The three descent-resolution steps

(21) The $k$-th column of $P$ is 2-pure and the $(k+1)$-th column of $P$ is 1-pure.

For an arbitrary 12-table it can happen also that two mixed columns form a descent, but such a descent will never arise in our process.

For each of the three types of descents, we will define what it means to resolve this descent. This is an operation which transforms the 12 -table $P$ by changing the entries in its $k$-th and $(k+1)$-th columns. These changes can be informally explained by Figure 2

For example, if $k$ is a descent of type (M1) in a 12-table $P$, then we define the 12-table $\operatorname{res}_{k} P$ as follows: the $k$-th column of $\operatorname{res}_{k} P$ is 1-pure; the $(k+1)$-th column of $\operatorname{res}_{k} P$ is mixed and the highest 2 in it is in the same row as the highest 2 in the $k$-th column of $P$; all other columns of $\operatorname{res}_{k} P$ are copied over from $P$ unchanged. The definitions of $\operatorname{res}_{k} P$ for the other two types of descents are similar. We say that $\operatorname{res}_{k} P$ is obtained from $P$ by resolving the descent $k$, and we say that passing from $P$ to $\operatorname{res}_{k} P$ constitutes a descent-resolution step. (Of course, a 12-table $P$ can have several descents and thus offer several ways to proceed by descent-resolution steps.)

Now the map B is defined as follows: take any 12-rpp $T$ and apply flip to it to get a 12-table flip $(T)$. Next, apply descent-resolution steps to flip $(T)$ in arbitrary order until we get a 12-table with no descents left. Put $\mathbf{B}(T):=P$.

In the full paper, it is proven that $\mathbf{B}(T)$ is well-defined (that is, the process terminates after a finite number of descent-resolution steps, and the result does not depend on the order of steps). It is also shown that $\mathbf{B}$ is an involution $\operatorname{RPP}^{12}(\lambda / \mu) \rightarrow \operatorname{RPP}^{12}(\lambda / \mu)$ that satisfies the claims of Lemma 3.5. An alternative proof of all these facts can be found in Section 6

\section{The classical Bender-Knuth involutions}

Fix a skew partition $\lambda / \mu$ and a positive integer $i$. We claim that the involution $\mathbf{B}_{i}: \operatorname{RPP}(\lambda / \mu) \rightarrow$ $\operatorname{RPP}(\lambda / \mu)$ we have constructed in the proof of Theorem 3.4 is a generalization of the $i$-th Bender-Knuth involution defined for semistandard tableaux. First, we shall define the $i$-th Bender-Knuth involution (following [GriRei15, proof of Proposition 2.11] and [Stan99, proof of Theorem 7.10.2]).

Let $\operatorname{SST}(\lambda / \mu)$ denote the set of all semistandard tableaux of shape $\lambda / \mu$. We define a map $\mathbf{B K}_{i}$ : $\operatorname{SST}(\lambda / \mu) \rightarrow \operatorname{SST}(\lambda / \mu)$ as follows:

Let $T \in \operatorname{SST}(\lambda / \mu)$. Then every column of $T$ contains at most one $i$ and at most one $i+1$. If a column contains both an $i$ and an $i+1$, we will mark its entries as "ignored". Now, let $k \in \mathbb{N}_{+}$. The $k$-th row of $T$ is a weakly increasing sequence of positive integers; thus, it contains a (possibly empty) string of $i$ 's 
followed by a (possibly empty) string of $(i+1)$ 's. These two strings together form a substring of the $k$-th row which looks as follows:

$$
(i, i, \ldots, i, i+1, i+1, \ldots, i+1) .
$$

Some of the entries of this substring are "ignored"; it is easy to see that the "ignored" $i$ 's are gathered at the left end of the substring whereas the "ignored" $(i+1)$ 's are gathered at the right end of the substring. So the substring looks as follows:

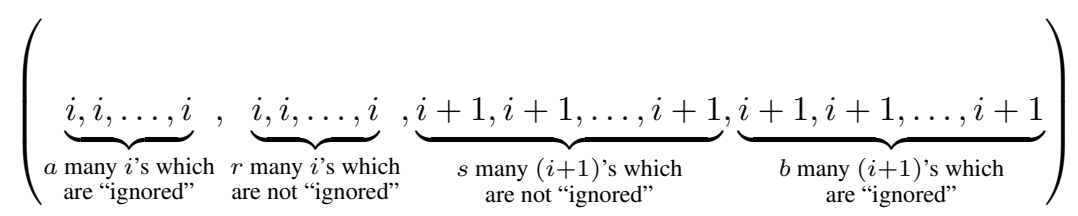

for some $a, r, s, b \in \mathbb{N}$. Now, we change this substring into

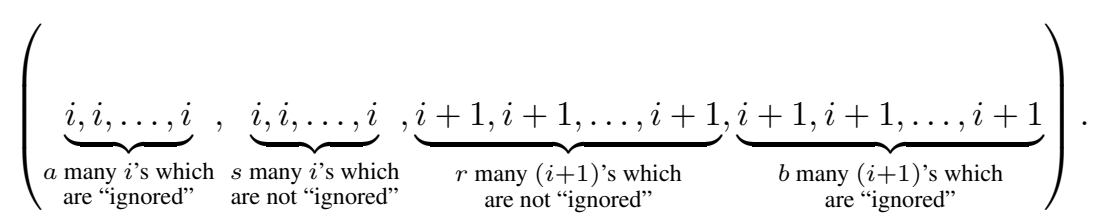

We do this for every $k \in \mathbb{N}_{+}$. At the end, we have obtained a new semistandard tableau of shape $\lambda / \mu$. We define $\mathbf{B K}_{i}(T)$ to be this new tableau.

Proposition 5.1. The map $\mathbf{B K}_{i}: \operatorname{SST}(\lambda / \mu) \rightarrow \operatorname{SST}(\lambda / \mu)$ thus defined is an involution. It is known as the $i$-th Bender-Knuth involution.

Now, every semistandard tableau of shape $\lambda / \mu$ is also an rpp of shape $\lambda / \mu$. Hence, $\mathbf{B}_{i}(T)$ is defined for every $T \in \operatorname{SST}(\lambda / \mu)$. Our claim is the following. The proof is straightforward from the definitions.

Proposition 5.2. For every $T \in \operatorname{SST}(\lambda / \mu)$, we have $\mathbf{B K}_{i}(T)=\mathbf{B}_{i}(T)$.

\section{The structure of 12-rpps}

In this section, we restrict ourselves to the two-variable dual stable Grothendieck polynomial $\widetilde{g}_{\lambda / \mu}\left(x_{1}, x_{2}, 0,0, \ldots ; \mathbf{t}\right)$ defined as the result of substituting $0,0,0, \ldots$ for $x_{3}, x_{4}, x_{5}, \ldots$ in $\widetilde{g}_{\lambda / \mu}$. We can represent it as a polynomial in $\mathbf{t}$ with coefficients in $\mathbb{Z}\left[x_{1}, x_{2}\right]$ :

$$
\widetilde{g}_{\lambda / \mu}\left(x_{1}, x_{2}, 0,0, \ldots ; \mathbf{t}\right)=\sum_{\alpha \in \mathbb{N}^{\mathbb{N}}+} \mathbf{t}^{\alpha} Q_{\alpha}\left(x_{1}, x_{2}\right),
$$

where the sum ranges over all weak compositions $\alpha$, and all but finitely many $Q_{\alpha}\left(x_{1}, x_{2}\right)$ are 0 .

We shall show that each $Q_{\alpha}\left(x_{1}, x_{2}\right)$ is either zero or has the form

$$
Q_{\alpha}\left(x_{1}, x_{2}\right)=\left(x_{1} x_{2}\right)^{M} P_{n_{0}}\left(x_{1}, x_{2}\right) P_{n_{1}}\left(x_{1}, x_{2}\right) \cdots P_{n_{r}}\left(x_{1}, x_{2}\right),
$$

where $M, r$ and $n_{0}, n_{1}, \ldots, n_{r}$ are nonnegative integers naturally associated to $\alpha$ and $\lambda / \mu$ and

$$
P_{n}\left(x_{1}, x_{2}\right)=\frac{x_{1}^{n+1}-x_{2}^{n+1}}{x_{1}-x_{2}}=x_{1}^{n}+x_{1}^{n-1} x_{2}+\cdots+x_{1} x_{2}^{n-1}+x_{2}^{n} .
$$


We fix the skew partition $\lambda / \mu$ throughout this whole section. We also make an assumption: namely, that the skew partition $\lambda / \mu$ is connected as a subgraph of $\mathbb{Z}^{2}$, and that it has no empty columns. This is a harmless assumption, since every skew partition $\lambda / \mu$ can be written as a disjoint union of such connected skew partitions and the polynomials $\widetilde{g}_{\lambda / \mu}$ get multiplied and the right hand side of 2 changes accordingly.

\subsection{Irreducible components}

We recall that a 12-rpp means an rpp whose entries all belong to the set $\{1,2\}$.

Given a 12-rpp $T$, consider the set $\operatorname{NR}(T)$ of all cells $(i, j) \in \lambda / \mu$ such that $T(i, j)=1$ but $(i+1, j) \in$ $\lambda / \mu$ and $T(i+1, j)=2$. Clearly, $\mathrm{NR}(T)$ contains at most one cell from each column; thus, let us write $\operatorname{NR}(T)=\left\{\left(i_{1}, j_{1}\right),\left(i_{2}, j_{2}\right), \ldots,\left(i_{s}, j_{s}\right)\right\}$ with $j_{1}<j_{2}<\cdots<j_{s}$. Because $T$ is a 12-rpp, it follows that the numbers $i_{1}, i_{2}, \ldots, i_{s}$ decrease weakly, therefore they form a partition which we denote

$$
\operatorname{seplist}(T):=\left(i_{1}, i_{2}, \ldots, i_{s}\right) \text {. }
$$

This partition will be called the seplist-partition of $T$.

We would like to answer the following question: for which partitions $\nu=\left(i_{1} \geq \cdots \geq i_{s}>0\right)$ does there exist a 12-rpp $T$ of shape $\lambda / \mu$ such that $\operatorname{seplist}(T)=\nu$ ?

A trivial necessary condition for this to happen is that there should exist some numbers $j_{1}<j_{2}<$ $\cdots<j_{s}$ such that

$$
\left(i_{1}, j_{1}\right),\left(i_{1}+1, j_{1}\right),\left(i_{2}, j_{2}\right),\left(i_{2}+1, j_{2}\right), \ldots,\left(i_{s}, j_{s}\right),\left(i_{s}+1, j_{s}\right) \in \lambda / \mu
$$

For each integer $i$, the set of all integers $j$ such that $(i, j),(i+1, j) \in \lambda / \mu$ is just an interval $\left[\mu_{i}+\right.$ $\left.1, \lambda_{i+1}\right]$, which we call the support of $i$ and denote $\operatorname{supp}(i):=\left[\mu_{i}+1, \lambda_{i+1}\right]$.

We say that a partition $\nu$ is admissible if every $k$ satisfies $\operatorname{supp}\left(i_{k}\right) \neq \varnothing$. (This is clearly satisfied when there exist $j_{1}<j_{2}<\cdots<j_{s}$ satisfying (3), but also in other cases.) Assume that $\nu=\left(i_{1} \geq \cdots \geq i_{s}>0\right)$ is an admissible partition. For two integers $a<b$, we let $\left.\nu\right|_{\subseteq[a, b)}$ denote the subpartition $\left(i_{r}, i_{r+1}, \ldots, i_{r+q}\right)$ of $\nu$, where $[r, r+q]$ is the (possibly empty) set of all $k$ for which $\operatorname{supp}\left(i_{k}\right) \subseteq[a, b)$. In this case, we pu(i) $\left.\# \nu\right|_{\subseteq[a, b)}:=q+1$, which is just the number of entries in $\left.\nu\right|_{\subseteq[a, b)}$. Similarly, we set $\left.\nu\right|_{\cap[a, b)}$ to be the subpartition $\left(i_{r}, i_{r+1}, \ldots, i_{r+q}\right)$ of $\nu$, where $[r, r+q]$ is the set of all $k$ for which $\operatorname{supp}\left(i_{k}\right) \cap[a, b) \neq \emptyset$.

We introduce several definitions: An admissible partition $\nu=\left(i_{1} \geq \cdots \geq i_{s}>0\right)$ is called

- non-representable if for some $a<b$ we have $\left.\# \nu\right|_{\subseteq[a, b)}>b-a$;

- representable if for all $a<b$ we have $\left.\quad \# \nu\right|_{\subseteq[a, b)} \leq b-a$;

a representable partition $\nu$ is called

- irreducible if for all $a<b$ we have $\left.\quad \# \nu\right|_{\subseteq[a, b)}<b-a$;

- reducible if for some $a<b$ we have $\left.\# \nu\right|_{\subseteq[a, b)}=b-a$.

Note that these notions depend on the skew partition; thus, when we want to use a skew partition $\widetilde{\lambda / \mu}$ rather than $\lambda / \mu$, we will write that $\nu$ is non-representable/irreducible/etc. with respect to $\widetilde{\lambda / \mu}$, and we denote the corresponding partitions by $\left.\nu\right|_{\subseteq[a, b)} ^{\widetilde{\lambda / \mu}}$.

(i) Here and in the following, $\# \kappa$ denotes the length of a partition $\kappa$. 
The motivation for these definitions is as follows: If a partition $\nu$ is non-representable, then there is no 12-rpp $T$ of shape $\lambda / \mu$ such that $\operatorname{seplist}(T)=\nu$. If $\nu$ is representable and $T$ is a 12-rpp of shape $\lambda / \mu$ such that $\operatorname{seplist}(T)=\nu$, then for any $a<b$ with $\left.\# \nu\right|_{\subseteq[a, b)}=b-a$, the columns $a, a+1, \ldots, b-1$ of $T$ are mixed columns whose entries are uniquely determined. So by the following Lemma, our problem reduces to looking at irreducible partitions.

Lemma 6.1. Let $\nu$ be a representable partition.

(a) There exist unique integers $\left(1=b_{0} \leq a_{1}<b_{1}<a_{2}<b_{2}<\cdots<a_{r}<b_{r} \leq a_{r+1}=\lambda_{1}+1\right)$ satisfying the following two conditions:

(a) For all $1 \leq k \leq r$, we have $\left.\# \nu\right|_{\subseteq\left[a_{k}, b_{k}\right)}=b_{k}-a_{k}$.

(b) The set $\bigcup_{k=0}^{r}\left[b_{k}, a_{k+1}\right)$ is minimal (with respect to inclusion) among all sequences $\left(1=b_{0} \leq\right.$ $\left.a_{1}<b_{1}<a_{2}<b_{2}<\cdots<a_{r}<b_{r} \leq a_{r+1}=\lambda_{1}+1\right)$ satisfying the above property.

Furthermore, for these integers, we have:

(b) The partition $\nu$ is the concatenation

$$
\left(\left.\nu\right|_{\cap\left[b_{0}, a_{1}\right)}\right)\left(\left.\nu\right|_{\subseteq\left[a_{1}, b_{1}\right)}\right)\left(\left.\nu\right|_{\cap\left[b_{1}, a_{2}\right)}\right)\left(\left.\nu\right|_{\subseteq\left[a_{2}, b_{2}\right)}\right) \cdots\left(\left.\nu\right|_{\cap\left[b_{r}, a_{r+1}\right)}\right)
$$

(where we regard a partition as a sequence of positive integers, with no trailing zeroes).

(c) The partitions $\left.\nu\right|_{\cap\left[b_{k}, a_{k+1}\right)}$ are irreducible with respect to $\lambda /\left.\mu\right|_{\left[b_{k}, a_{k+1}\right)}$, which is the skew partition $\lambda / \mu$ with columns $1,2, \ldots, b_{k}-1, a_{k+1}, a_{k+1}+1, \ldots$ removed.

Definition 6.2. In the context of Lemma 6.1 for $0 \leq k \leq r$ the subpartitions $\left.\nu\right|_{\cap\left[b_{k}, a_{k+1}\right)}$ are called the irreducible components of $\nu$ and the nonnegative integers $n_{k}:=a_{k+1}-b_{k}-\left.\# \nu\right|_{\cap\left[b_{k}, a_{k+1}\right)}$ are called their degrees. (For $T$ with seplist $(T)=\nu$, the $k$-th degree $n_{k}$ is equal to the number of pure columns of $T$ inside the corresponding $k$-th irreducible component. All $n_{k}$ are positive, except for $n_{0}$ if $a_{1}=1$ and $n_{r}$ if $b_{r}=\lambda_{1}+1$.)

\subsection{The structural theorem and its applications}

Recall that $\operatorname{RPP}^{12}(\lambda / \mu)$ denotes the set of all 12-rpps $T$ of shape $\lambda / \mu$, and let $\operatorname{RPP}^{12}(\lambda / \mu ; \nu)$ denote its subset consisting of all 12-rpps $T$ with $\operatorname{seplist}(T)=\nu$. Now we are ready to state a theorem that completely describes the structure of irreducible components:

Theorem 6.3. Let $\nu$ be an irreducible partition. Then for all $0 \leq m \leq \lambda_{1}-\# \nu$ there is exactly one 12-rpp $T \in \operatorname{RPP}^{12}(\lambda / \mu ; \nu)$ with $\# \nu$ mixed columns, $m$ 1-pure columns and $\left(\lambda_{1}-\# \nu-m\right)$ 2-pure columns. Moreover, these are the only elements of $\operatorname{RPP}^{12}(\lambda / \mu ; \nu)$. In other words, for an irreducible partition $\nu$ we have

$$
\sum_{T \in \operatorname{RPP}^{12}(\lambda / \mu ; \nu)} \mathbf{x}^{\operatorname{ircont}(T)}=\left(x_{1} x_{2}\right)^{\# \nu} P_{\lambda_{1}-\# \nu}\left(x_{1}, x_{2}\right) .
$$

After decomposing into irreducible components, we can obtain a formula for general representable partitions: 
Corollary 6.4. Let $\nu$ be a representable partition. Then

$$
\sum_{T \in \operatorname{RPP}^{12}(\lambda / \mu ; \nu)} \mathbf{x}^{\operatorname{ircont}(T)}=\left(x_{1} x_{2}\right)^{M} P_{n_{0}}\left(x_{1}, x_{2}\right) P_{n_{1}}\left(x_{1}, x_{2}\right) \cdots P_{n_{r}}\left(x_{1}, x_{2}\right),
$$

where the numbers $M, r, n_{0}, \ldots, n_{r}$ are defined above: $M=\# \nu, r+1$ is the number of irreducible components of $\nu$ and $n_{0}, n_{1}, \ldots, n_{r}$ are their degrees.

Note that the polynomials $P_{n}\left(x_{1}, x_{2}\right)$ are symmetric for all $n$. Since the question about the symmetry of $\widetilde{g}_{\lambda / \mu}$ can be reduced to the two-variable case, Corollary 6.4 gives an alternative proof of the symmetry of $\widetilde{g}_{\lambda / \mu}$ :

Corollary 6.5. The polynomials $\widetilde{g}_{\lambda / \mu} \in \mathbb{Z}\left[t_{1}, t_{2}, \ldots\right]\left[\left[x_{1}, x_{2}, x_{3}, \ldots\right]\right]$ are symmetric.

Another application of Theorem 6.3 is a complete description of the Bender-Knuth involutions on rpps we defined earlier.

Corollary 6.6. For a representable partition $\nu$, the map $\mathbf{B}: \operatorname{RPP}^{12}(\lambda / \mu ; \nu) \rightarrow \operatorname{RPP}^{12}(\lambda / \mu ; \nu)$ is the unique involution that interchanges the number of 1-pure columns with the number of 2-pure columns inside each irreducible component.

\section{References}

[FomGre06] Sergey Fomin, Curtis Greene, Noncommutative Schur functions and their applications, Discrete Mathematics 306 (2006) 1080-1096. doi:10.1016/S0012-365X(98)00140-X.

[Fulton97] William Fulton, Young Tableaux, London Mathematical Society Student Texts 35, Cambridge University Press 1997.

[GGL15] Pavel Galashin, Darij Grinberg, Gaku Liu, Refined dual stable polynomials and generalized Bender-Knuth involutions, October 15, 2015, arXiv:1509.03803v2

[GriRei15] Darij Grinberg, Victor Reiner, Hopf algebras in Combinatorics, August 25, 2015, arXiv:1409.8356v3 See also http://web.mit.edu/ darij/www/algebra/ HopfComb.pdf for a version which is more frequently updated.

[LamPyl07] Thomas Lam, Pavlo Pylyavskyy, Combinatorial Hopf algebras and K-homology of Grassmanians, arXiv:0705.2189v1. An updated version was later published in: International Mathematics Research Notices, Vol. 2007, Article ID rnm125, 48 pages. doi:10.1093/imrn/rnm125.

[Macdon95] Ian G. Macdonald, Symmetric Functions and Hall Polynomials, 2nd edition, Oxford University Press 1995.

[Stan99] Richard Stanley, Enumerative Combinatorics, volume 2, Cambridge University Press, 1999. 
